\title{
Poly-ADP-Ribosylation of Estrogen Receptor-Alpha by PARP1 Mediates Antiestrogen Resistance in Human Breast Cancer Cells
}

\author{
Nicholas Pulliam ${ }^{1,2}$, Jessica Tang ${ }^{2}$, Weini Wang ${ }^{2}$, Fang Fang ${ }^{2}$, Riddhi Sood ${ }^{3}$, \\ Heather M. O'Hagan ${ }^{2}{ }^{-D}$, Kathy D. Miller ${ }^{4}$, Robert Clarke ${ }^{5}$ and Kenneth P. Nephew ${ }^{1,2,6,7, *}$ \\ 1 Molecular and Cellular Biochemistry Department, Indiana University, Bloomington, IN 47405, USA; \\ npulliam@iu.edu \\ 2 Cell, Molecular and Cancer Biology Program, Medical Sciences Indiana University School of Medicine, \\ Bloomington, IN 47405, USA; jesstang@indiana.edu (J.T.); wang654@iu.edu (W.W.); ffang@indiana.edu (F.F.); \\ hmohagan@indiana.edu (H.M.O.) \\ 3 Department of Biology, Indiana University, Bloomington, IN 47401, USA; soodr@indiana.edu \\ 4 Department of Medicine, Indiana University School of Medicine, Indianapolis, IN 46202, USA; \\ kathmill@iu.edu \\ 5 Department of Oncology, Lombardi Comprehensive Cancer Center, Georgetown University, Washington, \\ DC 20057, USA; clarker@georgetown.edu \\ 6 Department of Cellular and Integrative Physiology, Indiana University School of Medicine, Indianapolis, \\ IN 46202, USA \\ 7 Department of Obstetrics and Gynecology, Indiana University School of Medicine, Indianapolis, \\ IN 46202, USA \\ * Correspondence: knephew@indiana.edu; Tel.: +1-812-855-9445
}

Received: 29 November 2018; Accepted: 2 January 2019; Published: 4 January 2019

\begin{abstract}
Therapeutic targeting of estrogen receptor- $\alpha(\mathrm{ER} \alpha)$ by the anti-estrogen tamoxifen is standard of care for premenopausal breast cancer patients and remains a key component of treatment strategies for postmenopausal patients. While tamoxifen significantly increases overall survival, tamoxifen resistance remains a major limitation despite continued expression of ER $\alpha$ in resistant tumors. Previous reports have described increased oxidative stress in tamoxifen resistant versus sensitive breast cancer and a role for PARP1 in mediating oxidative damage repair. We hypothesized that PARP1 activity mediated tamoxifen resistance in ER $\alpha$-positive breast cancer and that combining the antiestrogen tamoxifen with a PARP1 inhibitor (PARPi) would sensitize tamoxifen resistant cells to tamoxifen therapy. In tamoxifen-resistant vs. -sensitive breast cancer cells, oxidative stress and PARP1 overexpression were increased. Furthermore, differential PARylation of ER $\alpha$ was observed in tamoxifen-resistant versus -sensitive cells, and ER $\alpha$ PARylation was increased by tamoxifen treatment. Loss of ER $\alpha$ PARylation following treatment with a PARP inhibitor (talazoparib) augmented tamoxifen sensitivity and decreased localization of both ER $\alpha$ and PARP1 to ER $\alpha$-target genes. Co-administration of talazoparib plus tamoxifen increased DNA damage accumulation and decreased cell survival in a dose-dependent manner. The ability of PARPi to overcome tamoxifen resistance was dependent on ER $\alpha$, as lack of $\mathrm{ER} \alpha$-mediated estrogen signaling expression and showed no response to tamoxifen-PARPi treatment. These results correlate ER $\alpha$ PARylation with tamoxifen resistance and indicate a novel mechanism-based approach to overcome tamoxifen resistance in ER+ breast cancer.
\end{abstract}

Keywords: breast cancer; estrogen receptor; tamoxifen; antiestrogen resistance; PARP inhibitor 


\section{Introduction}

Estrogen receptor- $\alpha(E R \alpha)$ is a nuclear receptor [1-3] that responds to estrogen stimulation by translocating to the nucleus and regulating the transcription of target genes, including ER $\alpha$ and genes involved in regulating tumor progression [4]. Nearly $70 \%$ of breast cancers express ER $\alpha$ and rely on estrogen binding for growth and promotion of tumorigenesis [4]. Many ER $\alpha+$ breast cancer patients are treated with the anti-estrogen tamoxifen, which binds to the receptor within the ligand-binding domain (LBD). Mechanistically, binding of tamoxifen to the receptor results in a conformational change, initially decreasing the capacity of ER $\alpha$ to engage target genes. Aside from the tamoxifen-ER $\alpha$ interaction, tamoxifen-induced oxidative damage was reported to be an integral component of its therapeutic efficacy [5-7], through increased reactive oxygen species (ROS), and blocking ROS activity reduced anti-estrogen sensitivity [8]. Although highly effective in ER+ breast cancers, many of these patients develop resistance to tamoxifen, relapse and eventually succumb to the disease, despite continued ER $\alpha$ expression in their tumors [9]. In this regard, a better underlying mechanisms mediating tamoxifen resistance is of foremost clinical significance [10].

Endocrine resistance evolves from many mechanisms, including altered epigenetic regulation [11-13], as well as post-translational modifications (PTMs) of ER $\alpha[10,14,15]$. Many PTMs modify the ability of $E R \alpha$ to interact with transcriptional cofactors and engage target gene sequences in response to tamoxifen therapy, shifting tamoxifen from an antagonist to an ER $\alpha$ agonist [15]. In this context, additional understanding of $\mathrm{ER} \alpha$ regulation may help identify novel approaches to overcome tamoxifen resistance in the presence of persistent ER $\alpha$ expression.

Poly (ADP-ribose) polymerase 1 (PARP1) is a nuclear enzyme [16] that regulates cell signaling and the DNA damage response by poly-ADP-ribosylating (PARylating) target proteins, including PARP1 itself $[17,18]$. A critical role for PARP1 in tumor progression through regulation of oxidative DNA damage has been demonstrated and indicated as a mechanism to overcome PARPi resistance $[19,20]$. Classically, clinical applications of PARP inhibitors (PARPi), such as talazoparib and veliparib, target BRCA-related cancers with defects in HR-mediated DNA repair pathways [21-23]. In addition to DNA damage, PARP1 regulates the activity of many nuclear receptors, including ER $\alpha$ [24]. It was revealed in a breast cancer model (MCF7, ER $\alpha$-positive) that in response to estradiol, ER $\alpha$ and PARP1 co-localize to ER $\alpha$-target genes to regulate their expression [25]. Furthermore, in vascular smooth muscle cells, ER $\alpha$ was demonstrated to be PARylated by PARP1 within the DNA binding domain (DBD), allowing for increased and maintained nuclear localization of ER $\alpha$ [26]. However, whether or not ER $\alpha$ is PARylated in breast cancer cells, and if PARylation correlates with tamoxifen response, is unknown.

Based on previous reports demonstrating elevated oxidative stress in ER $\alpha$-positive, tamoxifen resistant breast cancer $[5,8,27]$ and the role of PARP1 in responding to oxidative damage [28,29], we hypothesized that combining the antiestrogen tamoxifen with a PARPi would sensitize tamoxifen resistant, ER $\alpha$-positive breast cancer to tamoxifen therapy. We tested our hypothesis in a MCF7-based breast cancer cell model systems. Combining a PARPi with tamoxifen altered ER $\alpha$ PARylation and decreased ER $\alpha$-PARylation, and this was sufficient to overcome tamoxifen resistance in hormone-refractory, ER $\alpha$-positive breast cancer cells. Our results demonstrate a novel mechanism of resistance to antiestrogen therapy and support a therapeutic approach for overcoming endocrine-resistant, ER $\alpha$-positive breast cancer.

\section{Results}

\subsection{Survival of Tamoxifen-Sensitive and-Resistant Breast Cancer Cells Is Inhibited by Tamoxifen-Talazoparib Combination}

We observed that tamoxifen-resistant (MCF7-T) cells showed increased levels of oxidative damage (Figure 1A), as indicated by immunofluorescence staining for the oxidative-damage marker 8-hydroxyguanosine (8-oxoG), consistent with previous studies [27] and validated this observation by measuring basal levels of reactive oxygen species (ROS) in tamoxifen-sensitive versus -resistant cell lines. As indicated by immunofluorescence, we observed increased $(p<0.05)$ levels of ROS in MCF7-T 
cells compared to the MCF7 parental cells (Figure 1B). We and others have demonstrated that oxidative damage caused by ROS promotes PARP1 activation [28,29]. We measured PARP1 levels and activity (indicated by poly-(ADP)-ribosylation (PARylation)), using western blot and ELISA assays and observed that basal PARP1 levels and activity were higher in MCF7-T cells compared to MCF7 cells (Figure 1C and Supplemental Figure S1A), as well as active ERBB2 (pERBB2), a marker of tamoxifen resistance [30]. Furthermore, tamoxifen treatment increased $(p<0.05)$ PARP1 activity in both parental and resistant cell lines (Figure 1D; ELISA assay).
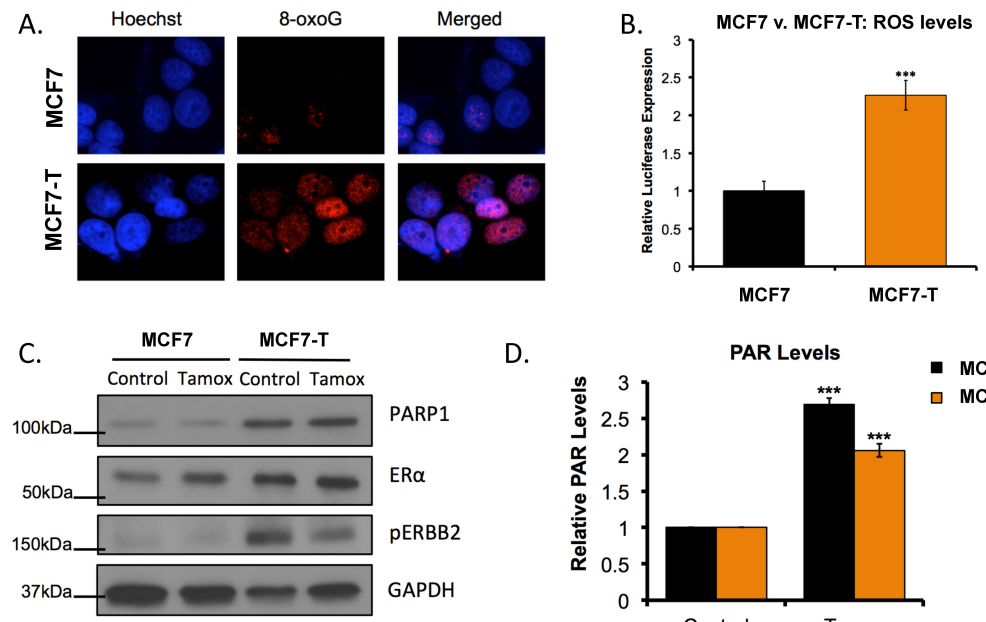

D.
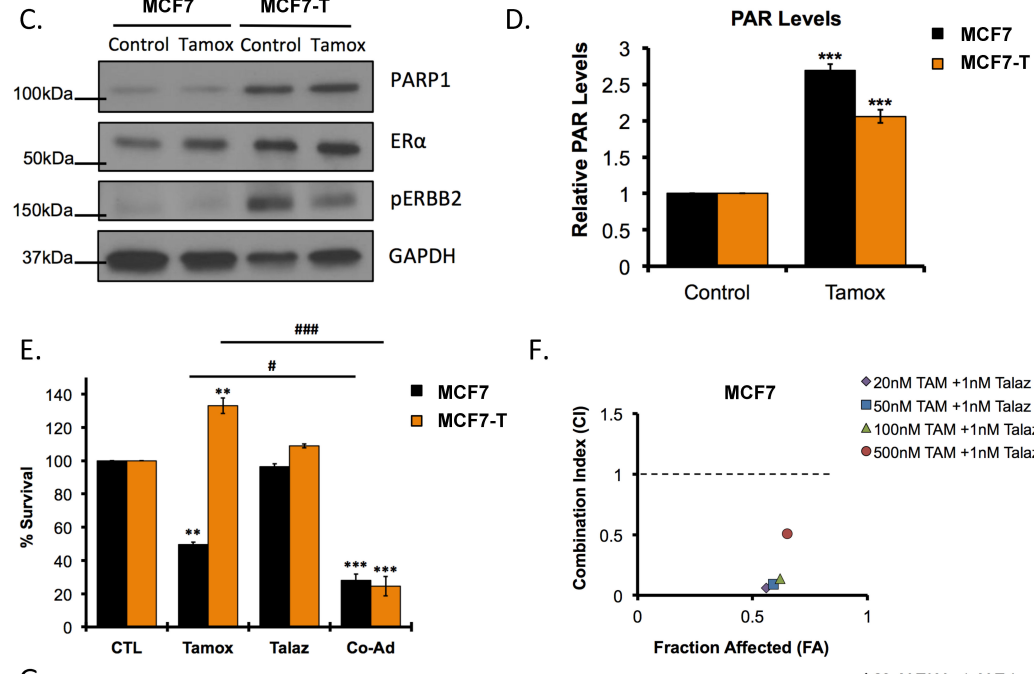

F.
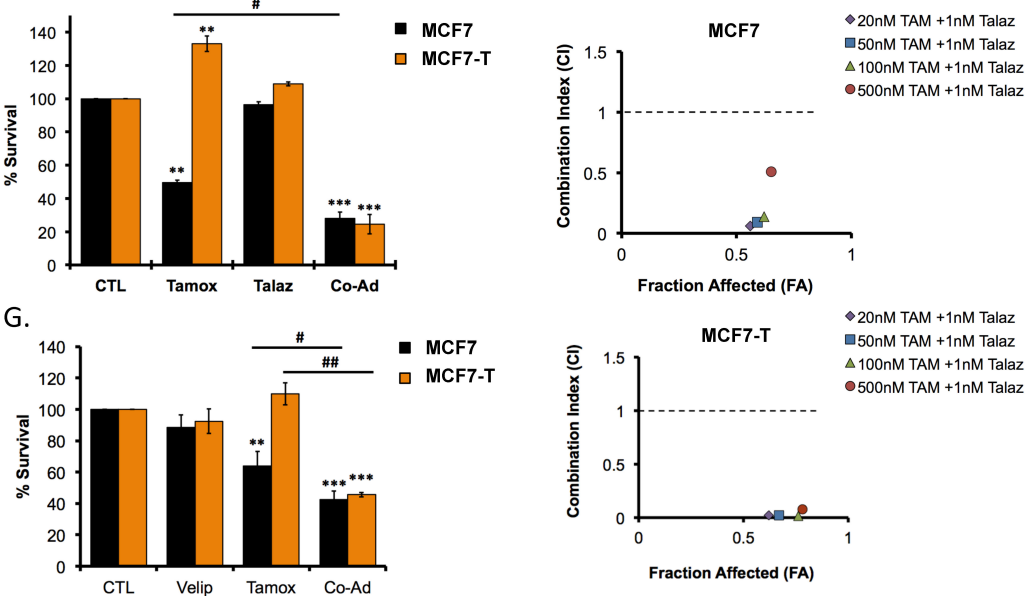

Figure 1. Therapeutic inhibition of PARP1 promotes sensitivity to tamoxifen treatment, in ER+ breast cancer, scale bar: $20 \mu \mathrm{m}$. (A) Immunofluorescence staining of 8-hydroxyguanosine (8-oxoG) in MCF7 and MCF7-T cell lines. (B) Basal ROS levels in MCF7 compared MCF7-T cells. Quantification is representative of at least three individual experiments. (C) MCF7 and MCF7-T cells were treated for $24 \mathrm{~h}$ with $100 \mathrm{nM}$ tamoxifen (Tamox) and western blot analysis performed against the indicated antibodies. (D) MCF7 and MCF7-T cells were treated with Tamox $(24 \mathrm{~h}, 100 \mathrm{nM})$ and subjected to PAR ELISA (E) MCF7 and MCF7-T cells were treated with $100 \mathrm{nM}$ Tamox or $1 \mathrm{nM}$ Talaz for $72 \mathrm{~h}$, alone and in combination, and colony formation assay was performed. (F) MCF7 (Top) and MCF7-T (Bottom) cells were treated with Tamox and Talaz for $72 \mathrm{~h}$, alone and in combination, and subjected to clonogenic survival assay to determine drug efficacy; $x$-axis is indicative of Fraction affected (FA), y-axis is indicative of the combination index (CI). Combinations beneath the black dashed line are synergistic. Results are representative of three independent experiments. (G) MCF7 and MCF7-T cells were treated with $100 \mathrm{nM}$ Tamox or $10 \mathrm{nM}$ veliparib (Velip) for $72 \mathrm{~h}$, alone and in combination, and colony formation assay was performed. PAR, Poly (ADP-ribose). ${ }^{* *} p<0.001,{ }^{* * *} p<0.0001$ compared to control, \# $p<0.01, \# \# p<0.001, \# \# p<0.0001$ relative to bracketed treatment. 
To examine whether PARP1 inhibition altered cell sensitivity to tamoxifen, we treated MCF7 and MCF7-T cells with tamoxifen alone or in combination with talazoparib and performed colony formation assays. As expected, tamoxifen alone decreased $(p<0.05)$ MCF7 clonogenic survival, and increased $(p<0.05)$ MCF7-T cell clonogenicity (Figure 1E). Despite differential response to tamoxifen, co-administration of tamoxifen and talazoparib decreased $(p<0.05)$ cell survival in both MCF7 and MCF7-T cells (Figure 1E, Supplemental Figure S1B,C). The observed decrease in colony formation was synergistic $(\mathrm{CI}<1)$ (Figure $1 \mathrm{~F}$, Supplemental Figure S1B,C), as determined by the Chou-Talalay method [31]. Similar combinatorial efficacy was observed upon co-administration of tamoxifen with the less potent PARPi veliparib (Velip; Figure 1G) [32].

To confirm the combinatorial efficacy of tamoxifen and talazoparib was not limited to the tamoxifen-resistant cells examined, we performed clonogenic survival assays in independently derived tamoxifen-resistant, ER $\alpha+$ breast cancer cell lines (LCC2, LCC9; ref [33]). Treatment of LCC2 and LCC9 with tamoxifen-talazoparib decreased $(p<0.05)$ cell survival $(\mathrm{CI}<1$; Supplemental Figure S1D,E, respectively). Furthermore, PARP1 activity was increased $(p<0.05)$ in LCC2 and LCC9 cell lines compared to MCF7 parental cells (Supplemental Figure S1F) and tamoxifen further increased $(p<0.05)$ PARP1 activity (Supplemental Figure S1G).

To validate the observed decrease in colony formation by MCF7 and MCF7-T cells in anchorage-dependent growth conditions, survival was also measured under anchorage-independent conditions. Both MCF7 and MCF7-T cells were plated within an agarose substrate and treated with tamoxifen in the presence and absence of talazoparib. Consistently, tamoxifen alone decreased $(p<0.05)$ MCF7 cell survival, while combination tamoxifen-talazoparib further decreased $(p<0.05)$ both MCF7 and MCF7-T survival compared to control or either single agent (Supplemental Figure S2A).

\subsection{Tamoxifen-Talazoparib Combinatorial Efficacy Is ER $\alpha$-Dependent}

To determine whether response to the combination was dependent on $E R \alpha$, we treated MDA-MB-231 cells (TNBC; negative for ER, PR and HER2) and MCF7-F (clonally derived, fulvestrant resistant and lack ER $\alpha$ signaling; [30]) with tamoxifen in the presence and absence of PARPi, and performed colony formation assays. In response to tamoxifen alone, no effect on cell survival was observed in either cell line (Supplemental Figure S2B-D), as expected. Similarly, following co-administration of tamoxifen with PARPi, no increase in cell response to tamoxifen treatment was observed. Fulvestrant treatment with or without talazoparib had no effect on MCF7-F cell survival (Supplemental Figure S2C,D).

In response to tamoxifen treatment, $\mathrm{ER} \alpha$ was shown to translocate to the nucleus in tamoxifen-resistant cells, with cytoplasmic localization being indicative of tamoxifen sensitivity [34]. In this regard, we observed tamoxifen treatment alone increased ER $\alpha$ nuclear localization (Supplemental Figure S3), consistent with previous reports [34], and co-administration with talazoparib increased both PARP1 and ER $\alpha$ cytoplasmic subcellular localization (caveolin-1 (Cav1) was used to validate decreased ER $\alpha$ function; [35]). Based on these results, it was next of interest to further examine a role for PARP1 in mediating tamoxifen resistance.

\subsection{Tamoxifen-Talazoparib Co-Administration Increases Cellular DNA Damage}

Inhibition of PARP1 using clinical PARPi increases DNA double strand break (DSB) repair in BRCA-proficient cells, which can be assessed by RAD51 foci formation [36]. To examine whether altered DSB repair contributed to talazoparib-mediated tamoxifen sensitivity, we measured RAD51 foci formation in both MCF7 and MCF7-T cells following treatment with talazoparib in the presence and absence of tamoxifen. In response to talazoparib treatment alone, we observed increased $(p<0.05)$ RAD51 foci in both MCF7 and MCF7-T cell lines (Figure 2A-C), consistent with the synthetic lethal relationship between PARP and BRCA-mediated DNA damage repair [21,22]. Interestingly, tamoxifen treatment alone did not alter RAD51 foci formation compared to control, and tamoxifen-talazoparib co-administration had no effect on RAD51 foci formation compared to talazoparib alone (Figure 2A-C). 
A.
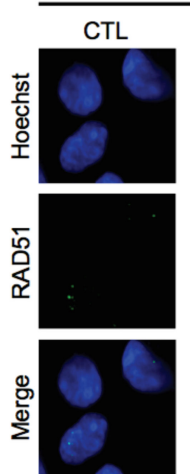

B.
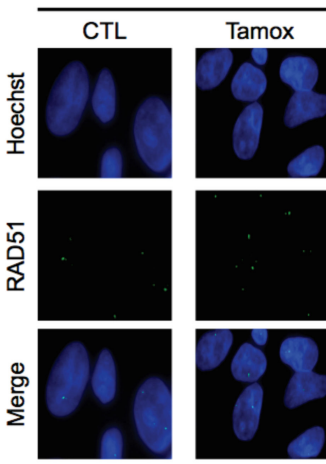

C.

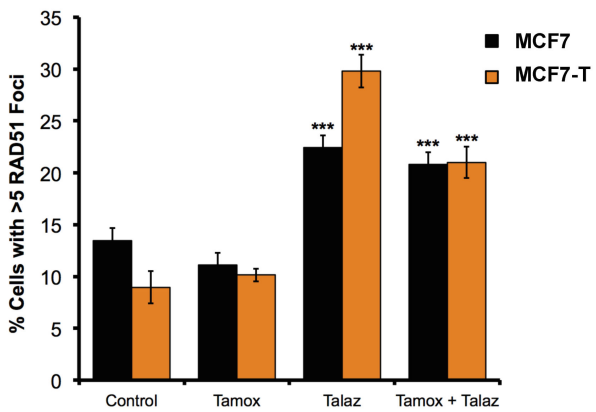

D.

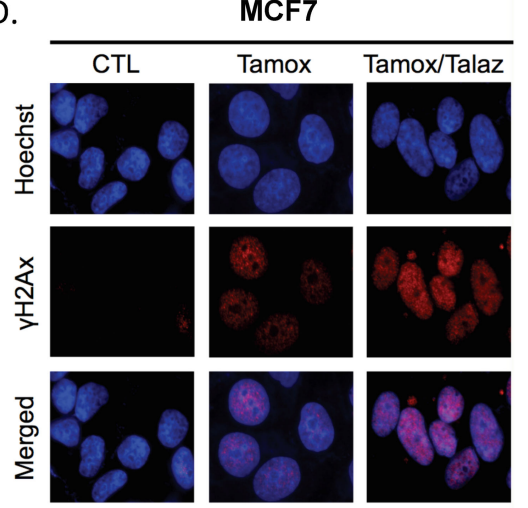

E.

MCF7-T
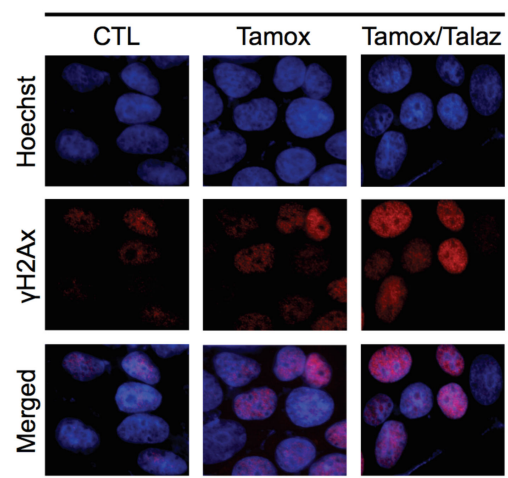

F.

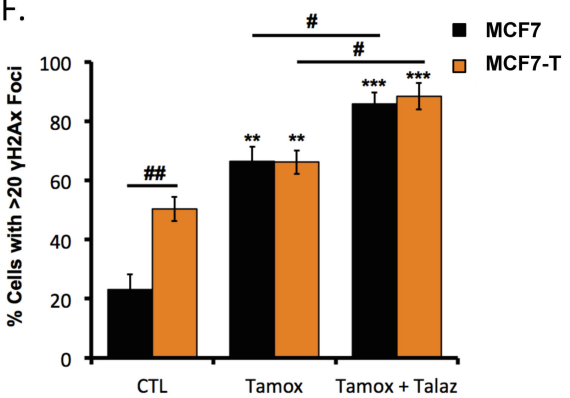

Figure 2. Tamoxifen-talazoparib co-administration induces DNA damage but not RAD51 foci formation. (A) MCF7 and (B) MCF7-T cells were treated with either $100 \mathrm{nM}$ tamoxifen (Tamox) or $1 \mathrm{nM}$ talazoparib (Talaz) for $72 \mathrm{~h}$, alone and in combination. $24 \mathrm{~h}$ post treatment RAD51 foci formation assay was performed. Scale bar: $20 \mu \mathrm{m}$. (C) Quantification is representative of three individual experiments. (D) MCF7 and (E) MCF7-T cells were treated with $100 \mathrm{nM}$ Tamox, with and without $1 \mathrm{nM}$ Talaz for $48 \mathrm{~h}$. $24 \mathrm{~h}$ post treatment cells immunofluorescence staining for $\gamma \mathrm{H} 2 \mathrm{AX}$ was performed. Scale bar: $20 \mu \mathrm{m}$. (F) Quantification is representative of three independent experiments. ${ }^{*} p<0.001$, ${ }^{* * *} p<0.0001$

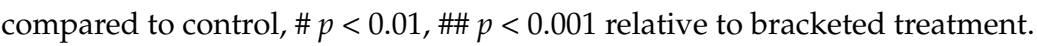

Because no specific effect on RAD51 was observed, we next examined DNA damage more generally. MCF7 and MCF7-T cells were treated with tamoxifen in the presence and absence of talazoparib, and immunofluorescence staining for $\gamma \mathrm{H} 2 \mathrm{Ax}$ foci formation was performed. In response to tamoxifen alone, we observed increased $(p<0.05) \gamma \mathrm{H} 2 \mathrm{Ax}$ staining, which was further increased $(p<0.05)$ by co-administration with talazoparib (Figure 2D-F). These findings are consistent with a previous study by Qi et al. showing that tamoxifen can impair homologous recombination repair, while promoting DNA damage accumulation [37]. 


\subsection{Tamoxifen Induces ROS, and ROS Promotes PARylaton of ER $\alpha$}

Because co-administration of tamoxifen and PARPi increased both tamoxifen response and $\gamma \mathrm{H} 2 \mathrm{AX}$ foci, but not RAD51 foci formation, it was of interest to further investigate the mechanism of PARPi-mediated tamoxifen sensitivity. We previously observed that basal ROS is increased in tamoxifen-resistant versus sensitive breast cancer cells (Figure 1A,B). Tamoxifen treatment increases intracellular ROS, and increased ROS is an important determinant of tamoxifen efficacy $[5,8]$. Based on these reports and our observations (Figure 2D-F), we tested whether tamoxifen increased oxidative damage and if tamoxifen-mediated ROS increased PARP1 activity. MCF7 and MCF7-T cells were treated with increasing concentrations of tamoxifen, and ROS levels were measured. We observed that tamoxifen increased $(p<0.05)$ ROS in a dose-dependent manner (Figure 3A), consistent with previous reports [5,8]. Furthermore, MCF7 and MCF7-T cells were treated with tamoxifen in the presence and absence of the ROS scavenger N-acetyl-L-cysteine (NAC) and cells were subjected to both immunofluorescence staining against 8-oxoG and western blot analysis. In response to tamoxifen, increased 8-oxoG staining was observed, which diminished upon co-administration with NAC (Figure 3B). Western blot analysis revealed increased PAR levels following tamoxifen treatment (Figure 3C and shown in Figure 1D), and co-administration with NAC decreased $(p<0.05)$ PAR levels (Figure 3C). Taken together, these data suggest tamoxifen increases PARP1 activity in a ROS-dependent manner.

Upon ROS-induced activation, PARP1 PARylates associated proteins to regulate cellular response to oxidative damage [28]. Moreover, in a vascular smooth muscle cell model [26], it was demonstrated that PARP1 PARylated ER $\alpha$, resulting in altered ER $\alpha$ nuclear translocation, response to ligand and transcriptional activity. PARP1 activity has been shown to be an important regulator of ER $\alpha$ function [26]; however, whether ER $\alpha$ PARylation is involved in breast cancer and furthermore tamoxifen resistance has not been examined. To initially test if ER $\alpha$ was PARylated and whether ROS promoted ER $\alpha$ PARylation, we treated MCF7-T cells with $\mathrm{H}_{2} \mathrm{O}_{2}$ (2 mM for $3 \mathrm{~h}$ ) and subsequently immunoprecipitated ER $\alpha$. Following addition of $\mathrm{H}_{2} \mathrm{O}_{2}$ (oxidative damage conditions), we observed increased ER $\alpha$ PARylation and an increased ER $\alpha$-PARP1 interaction (Figure 3D). To examine whether $\mathrm{ER} \alpha$ was differentially PARylated in tamoxifen-sensitive versus -resistant breast cancer cells, ER $\alpha$ was immunoprecipitated in untreated MCF7 and MCF7-T cells, and PAR accumulation was examined by western blot analysis. Interestingly, we observed greater interaction between ER $\alpha$ and PAR in the tamoxifen-resistant MCF7-T cell line compared to the sensitive MCF7 cells (Figure 3E). These results demonstrate that oxidative damage is sufficient to promote ER $\alpha$ PARylation in breast cancer and correlate ER $\alpha$ PARylation with tamoxifen resistance. 
A. MCF7 v. MCF7-T: Tamox-induced ROS

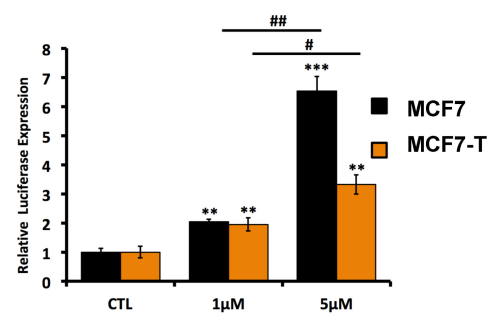

B.
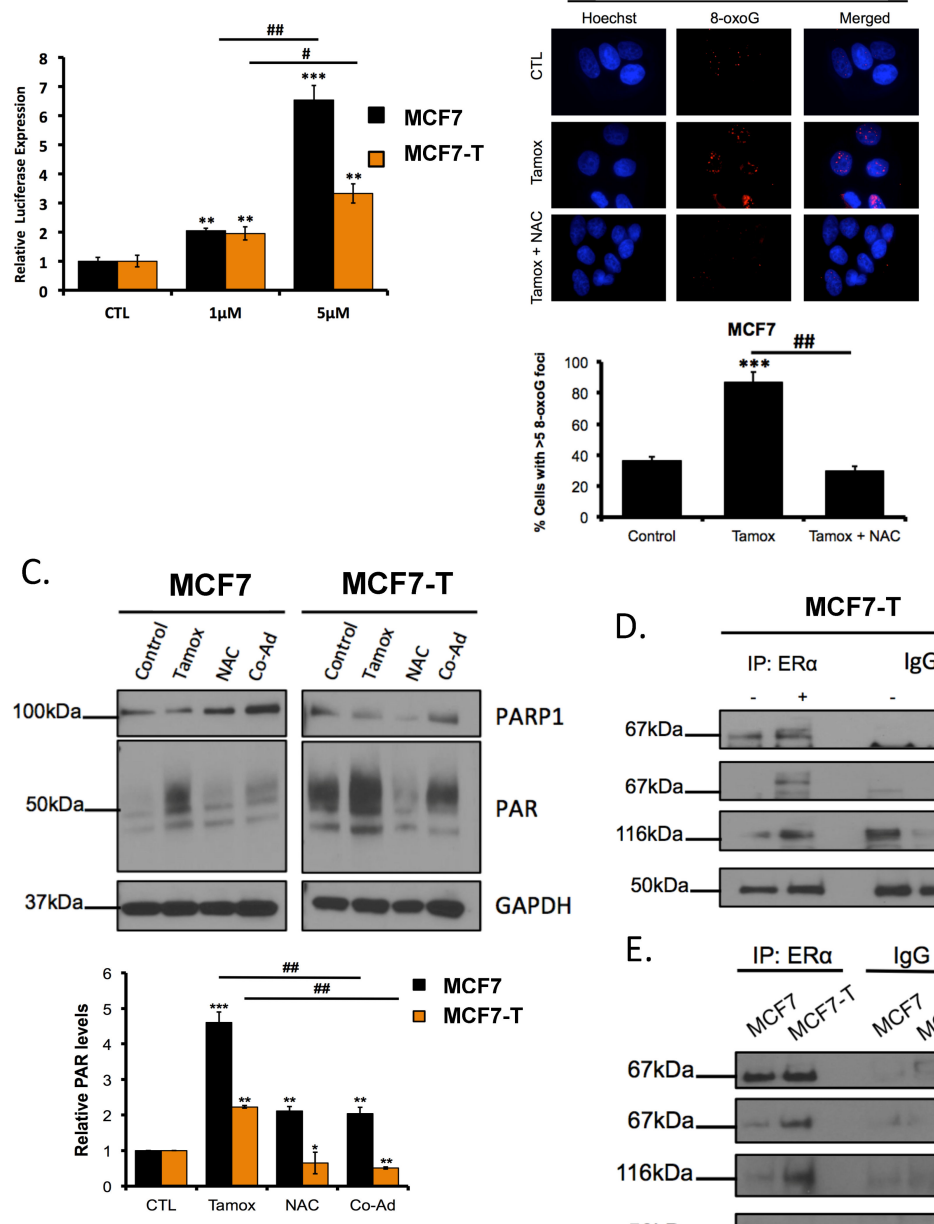

F.
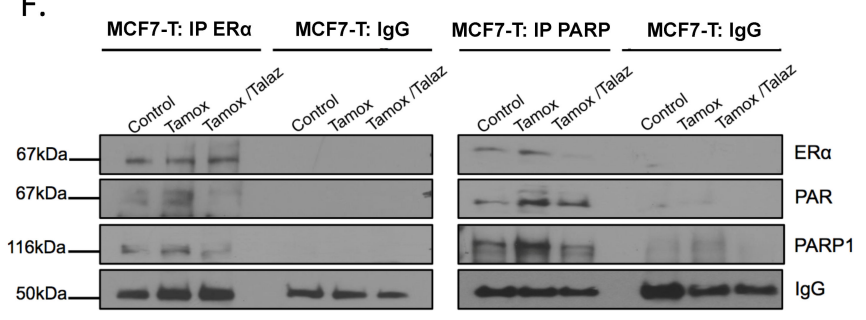

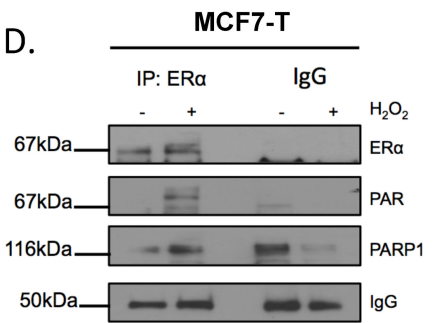

E.

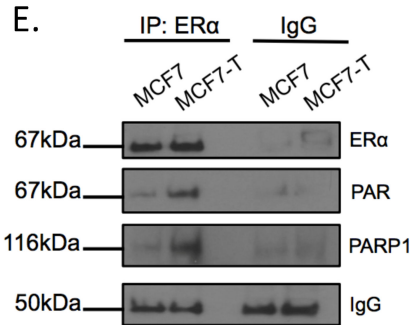

MCF7-T \pm Tamox

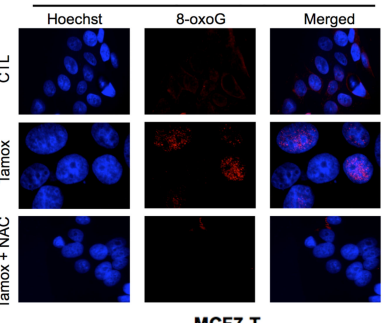

MCF7-T

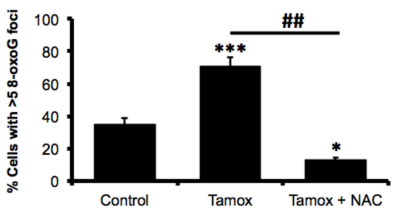

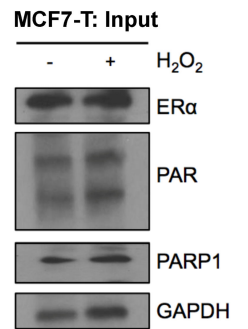

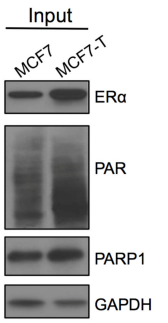

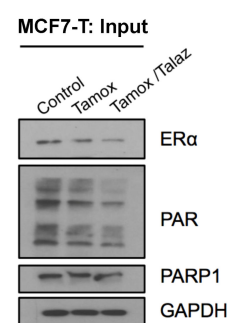

Figure 3. Tamoxifen-induced ROS accumulation promotes ER $\alpha$ PARylation. (A) MCF7 and MCF7-T cells were treated with the increasing concentrations of tamoxifen (Tamox) for $4 \mathrm{~h}$, and subjected to ROS assay. (B) MCF7 and MCF7-T cells were treated with Tamox $(1 \mu \mathrm{M})$ in the presence and absence of $1 \mathrm{mM}$ $\mathrm{N}$-acetyl-L-cysteine (NAC). $24 \mathrm{~h}$ post treatment immunofluorescence staining of 8-hydroxyguanosine (8-oxoG) was performed. Results are representative of three independent experiments, and quantified below. Scale bar: $20 \mu \mathrm{m}$. (C) MCF7 and MCF7-T cells were treated with $100 \mathrm{nM}$ Tamox with or without $1 \mathrm{mM}$ NAC (ROS scavenger). $24 \mathrm{~h}$ post treatment cell lysates were subjected to western blot analysis. (D) MCF7-T cells were treated with $2 \mathrm{mM} \mathrm{H}_{2} \mathrm{O}_{2}$ for $4 \mathrm{~h}$. Treated cells were subjected to immunoprecipitation $(\mathrm{ER} \alpha)$ and western blot analysis against the indicated antibodies. (E) MCF7 and MCF7-T subjected to immunoprecipitation (ER $\alpha)$ and western blot analysis against the indicated antibodies. (F) MCF7-T cells were treated for $4 \mathrm{~h}$ with $100 \mathrm{nM}$ Tamox with or without $1 \mathrm{nM}$ talazoparib (Talaz; pre-treat $24 \mathrm{~h}$ ). Post treatment cells subjected to immunoprecipitation and western blot analysis against the indicated antibodies. ${ }^{*} p<0.01,{ }^{* *} p<0.001,{ }^{* * *} p<0.0001$ compared to control, \# $p<0.01$, \#\# $p<0.001$ relative to bracketed treatment. 


\subsection{Tamoxifen Promotes PARylation of ER $\alpha$ and PARylation Mediates ER $\alpha$ Response}

We next hypothesized that tamoxifen-dependent ROS accumulation would likewise promote ER $\alpha$ PARylation. We treated both MCF7 and MCF7-T cells with tamoxifen, in the presence and absence of PARPi talazoparib and immunoprecipitated either ER $\alpha$ or PARP1 (MCF7-T cells). In response to tamoxifen treatment, we observed increased ER $\alpha$ PARylation in both MCF7 (Supplemental Figure S4A) and MCF7-T (Figure 3F) cells, and co-administration with talazoparib decreased the ER $\alpha$ PARylation, as well as the interaction with PARP1. Based on these results, we suggest that loss of ER $\alpha$ PARylation by PARPi treatment correlates with $\mathrm{ER} \alpha$ response to ligands and increased cell sensitivity to antiestrogen therapy.

$\mathrm{ER} \alpha$ is rapidly degraded in response to estradiol (E2) in breast cancer cells that are tamoxifen-responsive [30]. However, in tamoxifen resistant cells, E2 does not induce ER $\alpha$ degradation [30,33]. To validate that loss of PARP1 activity could mediate ER $\alpha$ response to ligand, we treated cells with E2 and performed western blot analysis. As a positive control, MCF7 cells were treated with E2 and reduced ER $\alpha$ levels were observed (Supplemental Figure S4B). In contrast, E2 did not decrease ER $\alpha$ protein levels in MCF7-T cells (Supplemental Figure S4C), whereas addition of the pure anti-estrogen fulvestrant markedly downregulated ER $\alpha$ protein levels (Supplemental Figure S4D). Next, MCF7-T cells were treated with talazoparib alone or in combination with E2, and under these conditions, a reduced level of ER $\alpha$ was observed (Supplemental Figure S4C). These results suggest that PARPi is able to regulate ER $\alpha$ response to ligand as a function of PARP1 activity, consistent with previous observations $[25,26]$.

\subsection{Talazoparib Reduces PARP1 and ER $\alpha$ Localization to ER $\alpha$ Target Genes}

Tamoxifen increased PARP1 activity, as well as ER $\alpha$ PARylation, and we observed that inhibition of PARP1 activity decreased nuclear ER $\alpha$ localization (Supplemental Figure S3). We then explored the role of PARP1 activity in regulating the enrichment of ER $\alpha$ at the promoter of ER $\alpha$ target genes. Based on $E R \alpha$-binding sites of previously identified ER $\alpha$ target genes, described by Jin et al., we performed PARP1 chromatin immunoprecipitation (ChIP) analysis against a panel of genes (RAR $\alpha$, MYC, GREB1, ERBB2, FOS, and XBP1) [4]. As a positive control, we also performed ER $\alpha$ ChIP against the same gene panel. As predicted, treatment with talazoparib in both the tamoxifen-sensitive (MCF7) and -resistant (MCF7-T) cell lines resulted in decreased $(p<0.05)$ recruitment of PARP1 to ER $\alpha$-binding sites in the majority of genes examined (Figure 4A,B). To a greater extent, ER $\alpha$ localization to ER $\alpha$-binding sites was likewise decreased $(p<0.05)$ following tamoxifen-talazoparib treatment relative to tamoxifen treatment alone (Figure 4C,D). These results suggest that the ER $\alpha$-PAR/PARP1 interaction is an important regulator of $\mathrm{ER} \alpha$-localization to $\mathrm{ER} \alpha$ target genes. 
A.

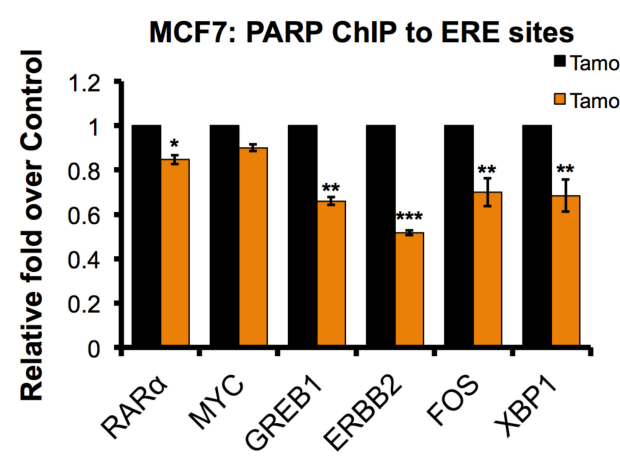

C.

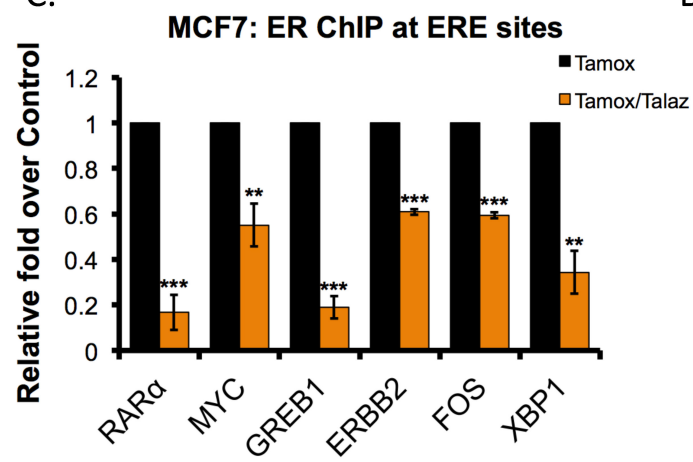

B.

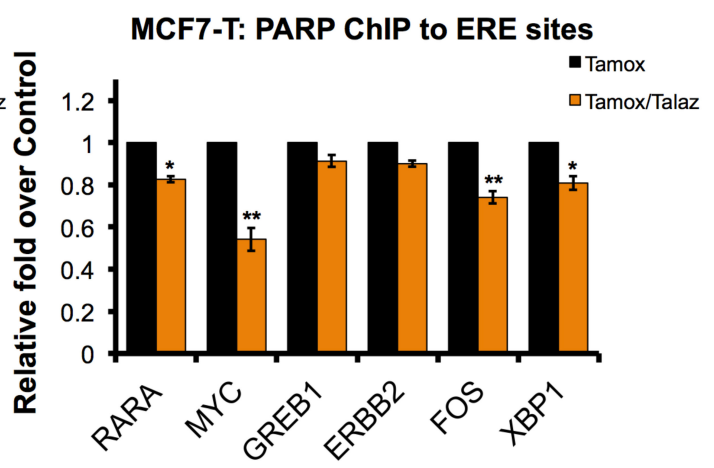

D.

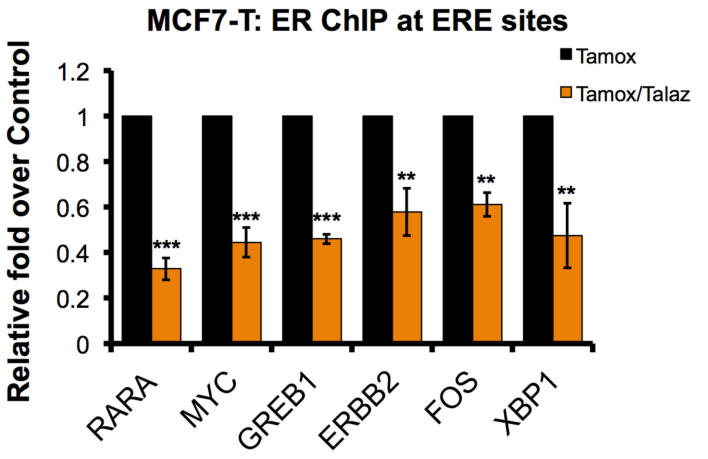

Figure 4. Talazoparib decreases PARP1 and ER $\alpha$ localization to ER $\alpha$-target genes. Chromatin immunoprecipitation (ChIP) analysis of PARP1 localization to ER $\alpha$-target genes (RARA, MYC, GREB1, ERBB2, FOS, XBP1) was performed in (A) MCF7 or (B) MCF7-T cells following tamoxifen treatment (Tamox; $100 \mathrm{nM}$ ) with or without talazoparib (Talaz; $1 \mathrm{nM}$ ). Chromatin immunoprecipitation (ChIP) analysis of ER $\alpha$ localization to ER $\alpha$-target genes (RARA, MYC, GREB1, ERBB2, FOS, XBP1) was performed in (C) MCF7 or (D) MCF7-T cells following Tamox treatment (100 nM) with or without 1 nM Talaz. ${ }^{*} p<0.01,{ }^{* *} p<0.001,{ }^{* * *} p<0.0001$ compared to control.

\subsection{Overexpression of miR-222 in Tamoxifen Resistant Cells Promotes PARP1 Activation}

While we demonstrated that tamoxifen increased ER $\alpha$ PARylation, we also observed that the basal ER $\alpha$ PARylation was increased in tamoxifen resistant versus sensitive cells (Figure 3E). As such, it was of interest to investigate the mechanism by which PARP1 was overexpressed in tamoxifen resistant breast cancer cells. It was recently demonstrated that microRNA-222 (miR-222) can both increase PARP1 activity, and promote PARPi sensitivity in ovarian cancer cells [38]. We and others demonstrated that miR-222 is overexpressed in tamoxifen resistant breast cancer cells compared to their sensitive counterparts [39-41]. To validate that miR-222 was overexpressed in tamoxifen resistant breast cancer, we measured basal miR-222 expression in parental MCF7 cells compared to MCF7-T by qRT-PCR analysis. We observed increased ( $p<0.05 ; \sim 6.5$-fold) miR-222 expression in tamoxifen resistant cells compared to the parental MCF7 cell line (Figure 5A). It was next of interest to determine whether tamoxifen could increase miR-222 expression and to test whether miR-222 overexpression was sufficient to promote tamoxifen resistance. MCF7 cells were treated with tamoxifen and qRT-PCR analysis was performed. As expected, tamoxifen treatment increased $(p<0.05)$ miR-222 expression in a dose-dependent manner (Figure 5B). We then treated MCF7 cells with tamoxifen in the presence and absence of miR-222 overexpression and subjected cells to clonogenic survival assays. In the absence of miR-222 overexpression, a dose-dependent decrease $(p<0.05)$ in cell survival was observed, and subsequent addition of miR-222 prevented tamoxifen-mediated cell death (Figure 5C). Consistently, we observed decreased $(p<0.05)$ cell survival in MCF7-T cells upon inhibition of miR-222 and treatment with tamoxifen (Supplemental Figure S5 and Figure 5D). 
A.

MCF7 v. MCF7-T: miR-222 Expression

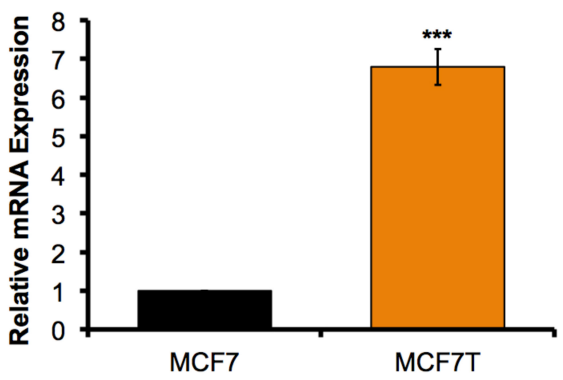

C.

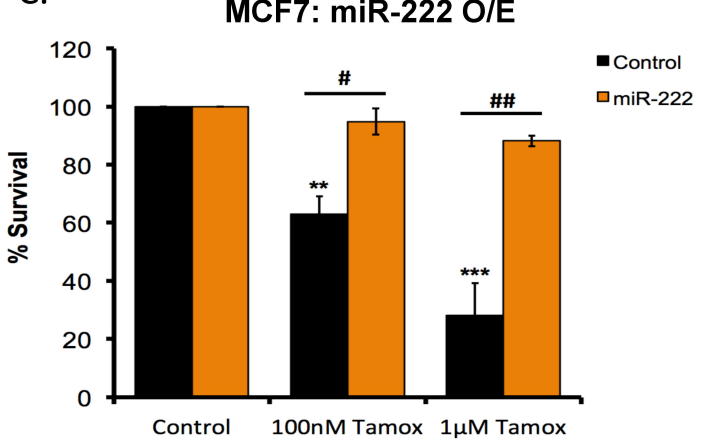

B. MCF7 v. MCF7-T: miR-222 Expression \pm Tamox

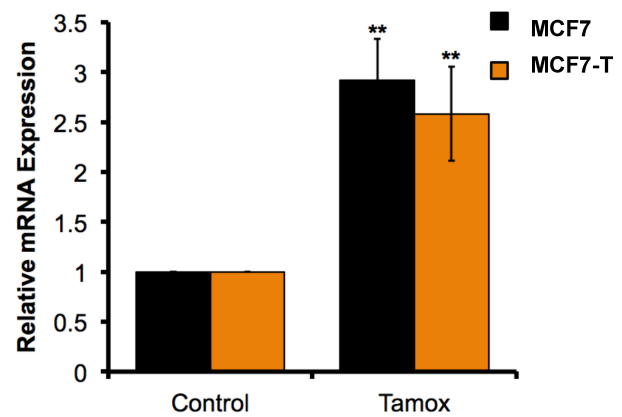

D. MCF7-T: Tamox \pm miR-222 Inhibitor

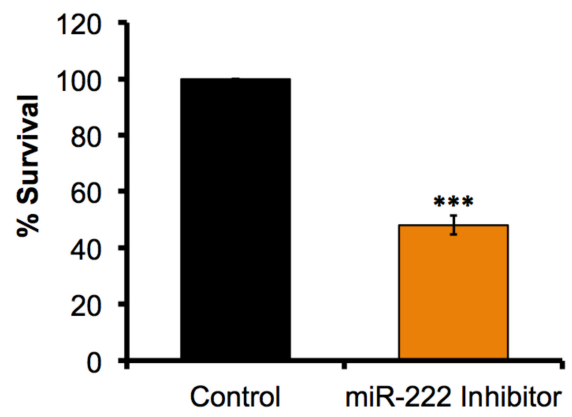

E. MCF7-T: Tamox \pm miR-222 Inhibitor

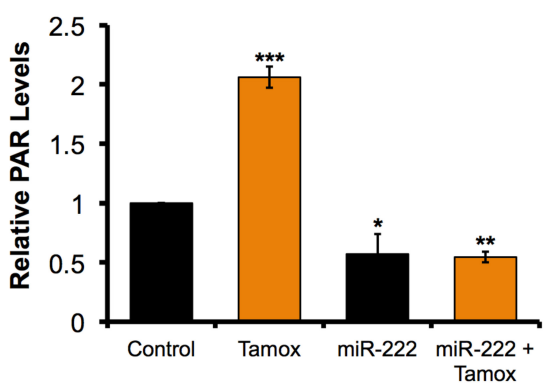

F.

MCF7: miR-222 O/E
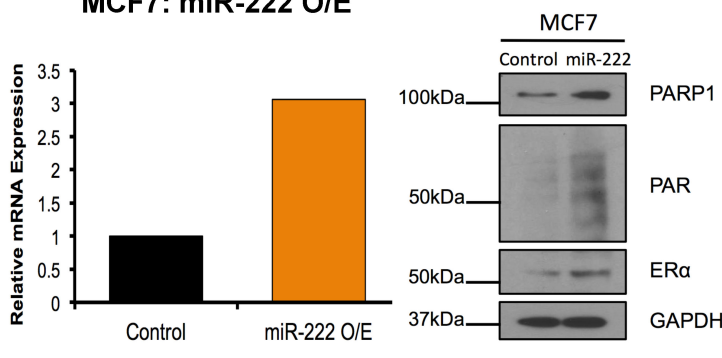

Figure 5. miR-222 mediates ER response to tamoxifen, and tamoxifen-mediated PARP1 activation. (A) Basal miR-222 expression was measured in MCF7 and MCF7-T cells by qRT-PCR analysis. (B) MCF7 and MCF7T cells were treated for $4 \mathrm{~h}$ with $100 \mathrm{nM}$ tamoxifen (Tamox) and miR-222 expression was measured by qRT-PCR analysis. (C) MC7 cells were treated with the indicated concentration of Tamox for $4 \mathrm{~h}$, with or without miR-222 overexpression. Treated cells were subjected to colony formation assays. Quantification is representative of three independent experiments. (D) MCF7-T cells were treated $72 \mathrm{~h}$ with $100 \mathrm{nM}$ Tamox with or without miR-222 inhibition (KD). Treated cells were subjected to colony formation assays. Quantification is representative of three independent experiments. (E) MCF7-T cells were treated for $4 \mathrm{~h}$ with $100 \mathrm{nM}$ Tamox with or without miR-222 inhibition. Post treatment cells were subjected to PAR ELISA. Quantification is representative of three independent experiments. (F) miR-222 was overexpressed in MCF7 cells and cell lysates subjected to qRT-PCR and western blot analysis. ${ }^{*} p<0.01,{ }^{* *} p<0.001,{ }^{* * *} p<0.0001$ compared to control, $\# p<0.01, \# \# p<0.001$ relative to bracketed treatment.

Given that tamoxifen was sufficient to increase both PARP1 activity and miR-222 expression, we determined whether tamoxifen-mediated PARP1 activation was miR-222 dependent. MCF7 cells were treated with tamoxifen in the presence and absence of miR-222 inhibitor and measured PAR levels. 
We observed that tamoxifen treatment increased $(p<0.05)$ PARP1 activity, as previously demonstrated, and subsequent inhibition of miR-222 decreased tamoxifen-mediated PARP1 activation (Figure 5E).

Regulation of PARP1 by miR-222 has been studied in depth in ovarian cancer cells [38]; however, whether miR-222 is sufficient to increase PARP1 activity in breast cancer is unknown. Using a miR-222 mimic, we exogenously expressed miR-222 in MCF7 tamoxifen-sensitive cells and measured PARP1 and PAR levels by western blot analysis. qRT-PCR validated overexpression of miR-222 $(p<0.05 ; \sim 3$-fold) (Figure 5F). Furthermore, overexpression of miR-222 increased both PARP1 and PAR levels (Figure 5F).

\section{8. miR-222 Regulates the Response to Tamoxifen-PARPi Co-Administration}

Because miR-222 overexpression increased PARP1 activity (Figure 5F), it was of interest to measure the effect on RAD51 foci formation. Consistent with our observation (Figure 2A-C), talazoparib treatment increased $(p<0.05)$ RAD51 foci in both MCF7 and MCF7-T cells (Figure 6A,B). Additionally, overexpression of miR-222 blocked formation of talazoparib-mediated RAD51 foci, irrespective of tamoxifen sensitivity (Figure 6A,B). We next investigated whether miR-222 mediated the PARPi response and, more importantly, the response to tamoxifen-talazoparib co-administration. First, we treated MCF7 cells with PARPi alone or combination with tamoxifen, in the presence and absence of miR-222 overexpression, and subjected cells to colony formation assays. In MCF7 cells overexpressing miR-222, we observed increased $(p<0.05)$ sensitivity to PARPi treatment (Figure 6C). Second, to determine whether miR-222 mediated the tamoxifen-talazoparib response, MCF7-T cells were treated with tamoxifen and increasing concentrations of talazoparib, with or without miR-222 expression. As miR-222 loss promoted sensitivity to tamoxifen (Figure 5D), tamoxifen-treated cells were used as the control (Figure 6D). In the presence of miR-222 (control), we observed a dose-dependent decrease in cell survival following tamoxifen-talazoparib co-administration; however, miR-222 inhibition diminished tamoxifen-talazoparib mediated cell death (Figure 6D). Taken together, our results support a role for talazoparib-mediated tamoxifen response in ER+, tamoxifen-resistant breast cancer. 
A.
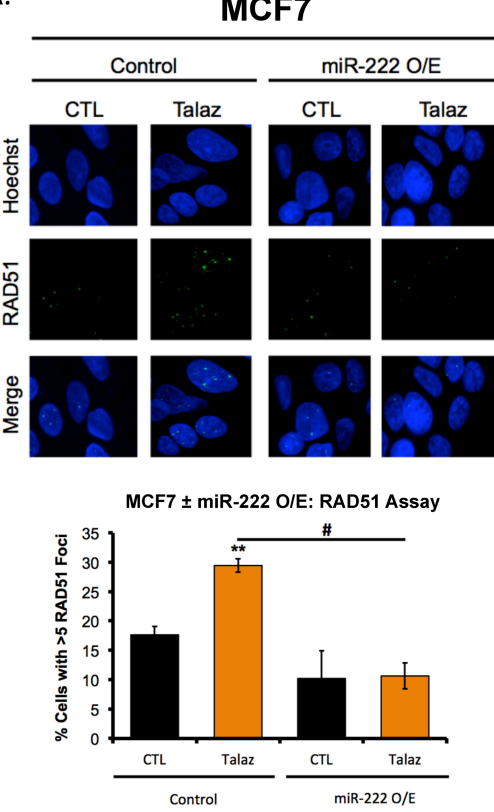

C.

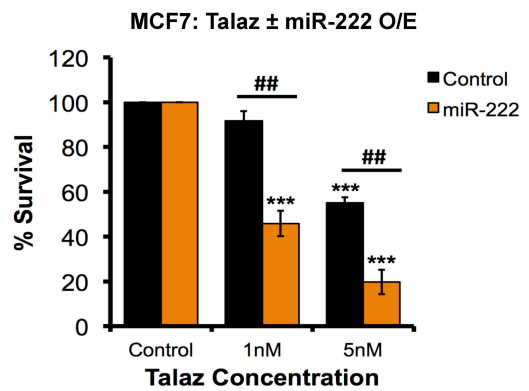

B.

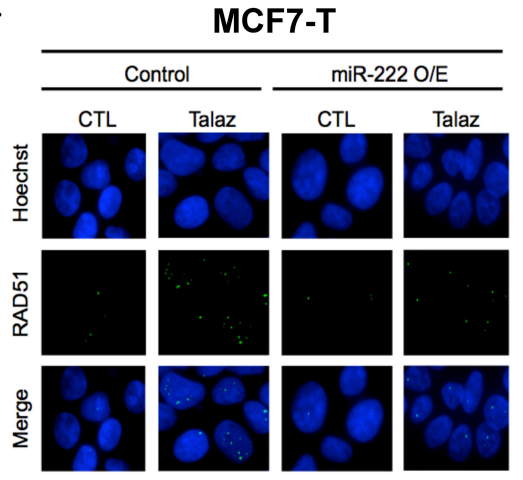

MCF7-T \pm miR-222 O/E: RAD51 Assay
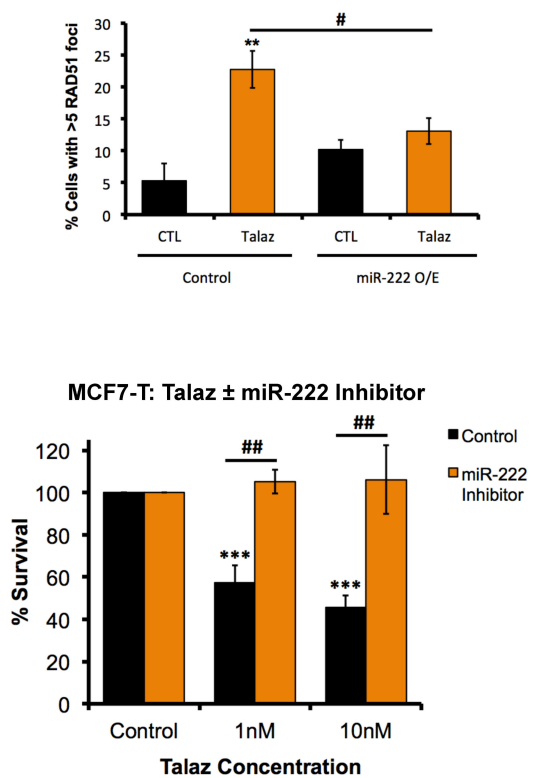

Figure 6. Tamoxifen-induced miR-222 expression mediates sensitivity to tamoxifen-talazoparib co-administration. (A) MCF7 and (B) MCF7-Tcells were treated with $1 \mathrm{nM}$ talazoparib (Talaz) for $48 \mathrm{~h}$ in the presence or absence of miR-222 overexpression (O/E). Post treatment cells were subjected to RAD51 foci formation assay. Quantification is representative of three independent experiments. Scale bar: $20 \mu \mathrm{m}$. (C) MCF7 cells were treated with increasing concentrations of Talaz for $48 \mathrm{~h}$, with or without miR-222 overexpression. Post treatment cells were subjected to colony formation assays. Quantification is representative of three independent experiments. (D) MCF7T cells were treated with $100 \mathrm{nM}$ tamoxifen (Tamox) and increasing concentration of Talaz for $48 \mathrm{~h}$, with or without miR-222 inhibition. Post treatment cells were subjected to colony formation assays. Quantification is representative of three independent experiments. ${ }^{* *} p<0.001,{ }^{* * *} p<0.0001$ compared to control, $\# p<0.01, \# \# p<0.001$ relative to bracketed treatment.

\section{Discussion}

Most breast tumors express $\mathrm{ER} \alpha$, and stimulation of $\mathrm{ER} \alpha$ by estradiol plays a significant role in breast tumor progression ER $\alpha$-targeted therapies including tamoxifen demonstrate exceptional clinical efficacy in treating breast cancer [42]. However, due to long-term treatment, resistance to tamoxifen therapy is common, even though most resistant tumors continue to express ER $\alpha$ [4]. Mechanisms of resistance include epigenetic alterations [11,41] and post-translational modifications [14], which alter the antagonistic effects of tamoxifen on ER $\alpha$ function. Attempts to overcome tamoxifen resistance through development of novel therapies that target estrogen synthesis $[15,43,44]$, and combination therapies have met with limited success, signifying a need to further explore therapeutic options to overcome tamoxifen resistance. In this regard, our study reveals that both oxidative damage and 
PARP1 levels are elevated in ER $\alpha+$ tamoxifen resistant breast cancer. Addition of a PARPi increases cell response to tamoxifen therapy in an ER $\alpha$-dependent manner. Through in vitro analysis, we show that combining tamoxifen with talazoparib increases both DNA damage and PAR accumulation and decreases survival of tamoxifen-resistant breast cancer cells. We identify miR-222 as a mediator of tamoxifen and PARPi response as well as the efficacy of tamoxifen-talazoparib combination. The results of this study support further investigation into combining antiestrogen with PARPi as a potential therapeutic option to overcome tamoxifen resistance in ER $\alpha+$ breast cancer.

Elevated levels of oxidative stress in tamoxifen resistant breast cancer have been previously reported [27], which we postulate may correlate with increased PARP1 expression and activity, implicating PARP1 as a therapeutic target to overcome tamoxifen resistance $[19,20]$. With respect to our overall hypothesis, we demonstrate that intracellular ROS levels are elevated in tamoxifen resistant versus sensitive cells, as are PARP1 levels and activity. Based on overexpression of PARP1, we show co-administration of PARPi with tamoxifen decreases cell survival in ER+ breast cancer cells. However, in breast cancer cells that do not depend on estrogen signaling for the survival (MDA-MB-231, MCF7-F), the combination had no effect, demonstrating that ER $\alpha$ expression is integral in mediating tamoxifen-talazoparib induced cell death. Additionally, treatment with the PARPi alone increases DSB break repair, as indicated by increased RAD51 foci formation. However, tamoxifen alone or in combination with PARPi had no effect on RAD51 foci formation. An increase in $\gamma \mathrm{H} 2 \mathrm{AX}$ following tamoxifen and tamoxifen-talazoparib treatment further supports the notion that tamoxifen-talazoparib induces DNA damage.

In addition to inhibiting ER $\alpha$ function, tamoxifen-induced intracellular ROS contributes to its therapeutic efficacy [5]. Interestingly, increased oxidative damage has been hypothesized to act as a mechanism to overcome PARPi resistance and a distinct therapeutic vulnerability in cancer cells $[19,45]$. In support of these studies, we demonstrate that tamoxifen treatment increases ROS accumulation in both MCF7 and MCF7-T cell lines, and tamoxifen-mediated ROS accumulation is sufficient to promote PARP1 activation. Inversely, ROS depletion following tamoxifen treatment diminishes PARP1 activity. We posit that tamoxifen treatment results in PARP1 activation and ultimately PARPi-tamoxifen response in a ROS-dependent manner.

Our results relate ROS accumulation by tamoxifen to increase ER $\alpha$ PARylation. PARP1 regulates cell signaling, including DNA damage response, through rapid PARylation of target proteins, including PARP1 itself [24]. In vascular smooth muscle cells, ER $\alpha$ is directly PARylated by PARP1, altering the ability of ER $\alpha$ to bind its target genes [26]. To our knowledge, this is the first demonstration that $E R \alpha$ is PARylated in breast cancer cells and that tamoxifen can induce ER $\alpha$ PARylation. Moreover, treatment with talazoparib diminishes ER $\alpha$ PARylation, as well as the interaction between ER $\alpha$ and PARP1, which correlates with talazoparib-mediated tamoxifen response. Differential ER $\alpha$ PARylation in tamoxifen sensitive versus resistant cells correlates with tamoxifen sensitivity. As ER $\alpha$ functions as a nuclear receptor and a transcription factor, talazoparib treatment decreases both PARP1 and $E R \alpha$ localization to ER $\alpha$-target genes, regardless of tamoxifen sensitivity. However, this observation is also consistent with decreased ER $\alpha$ levels following talazoparib treatment. Further experimentation will be necessary to understand the exact mechanism of talazoparib-mediated decrease in ER $\alpha$ localization to $\mathrm{ER} \alpha$-target genes.

In terms of a mediator of both tamoxifen and PARPi response, here we identify miR-222 as a potential negative regulator of tamoxifen-talazoparib mediated cell death. Tamoxifen resistant breast cancers overexpress miR-222 [39,40], and oxidative damage increases miR-222 expression. Interestingly, through inhibition of DSB damage repair, miR-222 overexpression is sufficient to promote PARPi sensitivity in ovarian cancer cells [38]. Beyond validating miR-222 overexpression in tamoxifen resistant cells, this is the first demonstration that upregulation of miR-222 increases PARP1 activation and PARPi sensitivity and decreases RAD51 foci formation in breast cancer cells. By inhibiting the oncomir (oncogenic miRNA), we further show the importance of miR-222 as a mediator of tamoxifen-talazoparib efficacy. Collectively, the data support a critical role for miR-222 in mediating 
response to tamoxifen-talazoparib treatment, with both loss and gain of miR-222 expression regulating response to single agent tamoxifen or talazoparib.

While the present study focuses on a proposed mechanism to overcome tamoxifen resistance through combination with PARPi, we do not believe this to be a mutually exclusive mechanism of induced cell death. In addition to PARPi-mediated tamoxifen response, our results also demonstrate a novel mechanism to overcome PARPi resistance, regardless of BRCA status, as all cell lines examined are BRCA-proficient and do not respond to PARPi as a single agent. Tamoxifen increases ROS and miR-222 expression but does not increase homologous recombination repair (RAD51 foci), all of which represent therapeutic avenues to increase sensitivity to PARPi $[19,38,45]$, suggesting a novel feedback loop such that both tamoxifen increases sensitivity to PARPi and PARPi to tamoxifen.

Additionally, work by our group and Muvarak et al. demonstrate that addition of talazoparib to cells promotes PARP1 trapping to DNA, increases DNA damage and ultimately cell death in combination with DNMT inhibitors [29,46]. ROS induces several classes of DNA damage to which PARP1 may localize, and talazoparib is the most potent PARP1 trapper [32]. It is conceivable that tamoxifen-mediated ROS accumulation and co-administration with talazoparib promotes trapping of PARP1 to DNA, enhancing the efficacy of tamoxifen-talazoparib co-administration. In support of this possibility, we demonstrate that tamoxifen co-administration with the PARPi veliparib, a less potent PARP-trapper, was able to induce cell death but not to the extent of the tamoxifen-talazoparib combination treatment [32]. Moreover, we show tamoxifen-talazoparib co-administration reduces nuclear localization of both ER $\alpha$ and PARP1, though some PARP1 remains in the nucleus (Supplemental Figure S3). Talazoparib robustly reduces ER $\alpha$ at ERE sites and PARP1 but to a much lesser extent (Figure 4), indicating that PARP1 may be more tightly bound to the DNA (albeit not directly explored in this study). It is important to note that differences in ChIP enrichment at ERE sites may be due to either altered ER $\alpha$ and PARP1 nuclear localization, as demonstrated (Supplemental Figure S3), or decreased DNA affinity in the absence of PARP1 activity [27]. Further studies are needed to discriminate between these mechanisms. Nonetheless, it seems reasonable to suggest that some of the enhanced response to tamoxifen-talazoparib co-administration is a result of tamoxifen-mediated ROS accumulation and PARP1 trapping.

In summary, using a model system based on the MCF7 breast cancer cell line, we show addition of a PARPi increases cell response to tamoxifen in tamoxifen-resistant ER $\alpha+$ breast cancer. We put forth a model of tamoxifen-mediated ROS accumulation, which increases miR-222 expression and subsequently PARP1 activity, priming the cell towards tamoxifen sensitivity, in an ER $\alpha$-dependent manner (Supplemental Figure S6) and in the context of intact BRCA. Collectively, these data support additional pre-clinical and clinical investigation of tamoxifen-talazoparib combination for patients with tamoxifen resistant $\mathrm{ER} \alpha+$ breast cancer. Our future studies aimed at better understanding the significance of ER $\alpha$ PARylation and increasing the translational impact of the therapeutic combination will include multiple cell lines and in vivo model systems.

\section{Materials and Methods}

\subsection{Cell Lines, Culture Conditions and Reagents}

MCF7 and anti-estrogen resistant derivatives (MCF7-T and MCF7-F) were maintained and established as we have previously described [30]. Briefly, MCF7-T cells were maintained in tamoxifen-containing media, except prior to drug treatment. The maintenance concentration of tamoxifen was $100 \mathrm{nM}$. MCF7/LCC2 and MCF7/LCC9 cells lines (anti-estrogen resistant) were derived and maintained as described by Brünner et al. [33]. Cell lines were authenticated in 2017 by ATCC and tested for mycoplasma contamination (Manassas, VA, USA). To ensure cell line integrity, all cell lines were thawed at frequent intervals and not used beyond 30 passages. Additionally, cell morphology was monitored for each cell line, and proper media and growth conditions selected $[29,30]$. Hydrogen peroxide $\left(\mathrm{H}_{2} \mathrm{O}_{2}\right)$ was purchased from EMD Millipore (Billerica, MA, USA). Tamoxifen (Tamox or 4-OHT) was purchased from Sigma Aldrich (St. Louis, 
MO, USA). Talazoparib (Talaz; PARPi) was provided by Pfizer/Medivation (San Francisco, CA, USA). Primary antibody dilutions were as followed: PARP1 (Cell Signaling, Danvers, MA, USA; 1:3000), PAR (Trevigen, Gaithersburg, MD, USA; 1:2000) ER $\alpha$ (Santa Cruz, Dallas, TX, USA; 1:2000), pERBB2 (Cell Signaling; 1:2000), GAPDH (Santa Cruz; 1:2000), IgG (Santa Cruz; 1:5000), $\beta$-Tubulin (Santa Cruz; 1:5000).

\subsection{Clonogenic Survival and MTT Assay}

Cells were seeded to $60-70 \%$ confluence in $10 \mathrm{~cm}$ plates and treated for the indicated times. $24 \mathrm{~h}$ following treatment, cells were washed, serially diluted, and plated in triplicate on 6-well plates (500-1000 cells/well). 6-14 days of cell growth were allowed for colony formation, followed by staining with $0.5 \%$ crystal violet. Cell counts were normalized to untreated controls as previously described [47]. Combination treatment scheme is as followed: Co-administration ('Co-ad') with both $100 \mathrm{nM}$ tamoxifen (Tamox) and $1 \mathrm{nM}$ talazoparib (Talaz) on day 1, and $100 \mathrm{nM}$ Tamox on days 2 and 3, unless otherwise indicated.

\subsection{RNA Extraction, Quantitative RT-PCR and Cell Transfection}

RNA was extracted via RNeasy kit (Qaigen, Venlo, Limburg, The Netherlands), and cDNA prepared with MMLV reverse transcriptase (Promega, Madison, WI, USA). qRT-PCR was performed as we have previously described [48], using EEF1A as the internal control. Cells were treated with either miR-222 mimic (Ambion, Carlsbad, CA, USA; 4464066) or miR-222 inhibitor (Ambion; AM17000) within the indicated treatments conditions and according to the manufacturer's protocol using the Lipofectamine 3000 reagent (Thermo Fisher Scientific, Waltham, MA, USA; L3000008).

\subsection{PAR-Capture ELISA}

Confluent (80\%) $10 \mathrm{~cm}$ plates were treated as described in the text. Following treatment, samples were harvested, $20 \%$ SDS was added (to a final concentration of $1 \%$ ), protein concentration measured according to the manufacturer's protocol (Trevigen; 4520-096-K) and the lysate was snap-frozen and placed at $-80^{\circ} \mathrm{C}$. Preparation of the PAR standard curve and treated samples was performed according to the manufacturer's protocol, and as we have described [29]. In brief, $100 \mu \mathrm{L}$ ( $20 \mu \mathrm{g}$ protein) of each sample was added to a 96-well plate, pre-coated with PAR capture antibody and allowed to incubate overnight. Wells were then washed with PBST (PBS + 0.1\% Tween 20) and incubated with primary antibody (PAR Polyclonal antibody), washed with PBST and incubated in secondary antibody (Goat Anti-Rabbit IgG HRP). Plates were washed with PBST, chemiluminescent substrate was added, and luminescence measured. The data are reported as the relative change in PAR concentration compared to control.

\subsection{ROS Assays}

Confluent $10 \mathrm{~cm}$ plates were treated as indicated in the text. Following treatment, $2 \times 10^{4}$ cells/well were plated on 96-well plates. $24 \mathrm{~h}$ post plating, ROS assay, measuring $\mathrm{H}_{2} \mathrm{O}_{2}$ levels, (Promega) was performed as previously described [47].

\subsection{Immunofluorescence}

Cells were treated as described and plated on glass slides (50,000 cells/well), fixed and permeabilized as described by Sakai et al. [49]. Following permeabilization, cells were incubated for 1 h with RAD51 antibody (1:1000), $\gamma$ H2Ax antibody (1:1000), or 8-hydroxyguanosine (8-oxoG) (1:1000). Secondary antibody (Invitrogen, Carlsbad, CA, USA) was added and slides incubated for $45 \mathrm{~min}$. Nuclei were counterstained with Hoechst stain (1:5000; 33342, Invitrogen). Images were captured using a Nikon NIE microscope, and processed using NIS Elements Viewer. Cells with 5 or more RAD51 
foci were considered positive (per ref [49]) and cells with 20 or more $\gamma \mathrm{H} 2 \mathrm{Ax}$ foci were considered positive. Results are representative of three independent experiments \pm SEM.

\subsection{Western Blot Analysis}

Total cell lysate was prepared with RIPA lysis buffer. Western blot analysis was performed as we have described previously [47].

\subsection{Combination Index and Synergism}

Cells were treated and plated as indicated for clonogenic survival assays (500 cells/well). Following treatment, percent survival subtracted from $100 \%$ was indicative of the fraction affected (FA). Subsequent combination indices, and synergism determination, were determined by the Chou-Talalay method ([31]; with mutually non-exclusive assumption) using CompuSyn Software [50].

\subsection{Immunoprecipitation Assays}

MCF7 and MCF7-T cells were grown to confluence (90\%) in $15 \mathrm{~cm}$ plates, and treated as described in the text. $24 \mathrm{~h}$ following treatment, cells were washed with PBS and harvested in immunoprecipitation (IP) lysis buffer and protease inhibitors. The lysate was sonicated briefly and rotated at $4{ }^{\circ} \mathrm{C}$, followed by centrifugation at $13,000 \mathrm{rpm}$ for $10 \mathrm{~min}$. The lysates were added to $50-60 \mu \mathrm{L}$ of pre-conjugated protein A/G beads with the indicated antibodies. Beads were pelleted $\left(1000 \mathrm{rpm}, 4{ }^{\circ} \mathrm{C}\right)$ and washed in IP buffer. The samples were run on SDS-PAGE gel and immunoblot analysis was performed against the indicated antibodies [4,47].

\subsection{Chromatin Immunoprecipitation (ChIP)- $q P C R$}

MCF7 and MCF7-T cells were grown to confluency (90\%) in $15 \mathrm{~cm}$ plates, and treated as described in the text. $24 \mathrm{~h}$ following treatment cells were washed with PBS, crosslinked with $10 \%$ formalin and harvested in immunoprecipitation (IP) lysis buffer. Following IP (previously described, [48]) with antibody pre-conjugated protein agarose beads, the crosslinks were reversed, and DNA was purified. ChIP-qPCR primers targeting the estrogen response element (ERE) were designed as previously described by Jin et al. [4]. A negative control region was used, as we have previously described [48].

\subsection{Statistical Analysis}

All data, unless noted otherwise, are represented as mean value \pm SEM of at least three biological replicates. $\mathrm{IC}_{50}$ data was determined by Prism 6 (GraphPad Software, San Diego, CA, USA), using logarithm normalized sigmoidal dose-curve fitting. Tukey's test for multiple comparisons correction was used to analyze the significance among different groups in biological assays, unless otherwise stated. For in vivo experiments, ANOVA/Mann-Whitney tests (GraphPad) were used to determine statistical significance, as described [46].

\section{Conclusions}

Therapeutic targeting of ER $\alpha$ by tamoxifen is standard of care for premenopausal breast cancer. While tamoxifen increases overall survival, tamoxifen resistance remains a major limitation, despite continued expression of ER $\alpha$ in resistant tumors. Towards a better molecular understanding of endocrine resistance, we used clonally derived ER $\alpha$-positive, antiestrogen-resistant breast cancer MCF7-cell models and demonstrated differential ER $\alpha$ PARylation in tamoxifen-resistant vs. -sensitive cells. We further demonstrated that tamoxifen treatment was sufficient to activate PARP and increase $E R \alpha$ PARylation in an ROS-dependent manner. Combination treatment of the PARP inhibitor talazoparib with tamoxifen effectively blocked ER $\alpha$ PARylation, markedly decreased ER $\alpha$ localization to ER $\alpha$ target genes and significantly enhanced response to tamoxifen in an ER $\alpha$-dependent manner in models of acquired tamoxifen resistance. We provide the first pre-clinical evidence that combining 
a PARP inhibitor with tamoxifen has significant activity against tamoxifen-resistant breast cancers which continue to express $\mathrm{ER} \alpha$, a therapeutic strategy that may warrant further investigation.

Supplementary Materials: The following are available online at http:/ /www.mdpi.com/2072-6694/11/1/43/s1, Figure S1: Tamoxifen-talazoparib co-administration decrease cell survival and increase PARP activity, Figure S2: Tamoxifen-talazoparib efficacy is ER $\alpha$-dependent, Figure S3: Tamoxifen and talazoparib alter ER $\alpha$ nuclear localization, Figure S4: Tamoxifen induces ER PARylation in tamoxifen-sensitive MCF7 cell line, Figure S5: miR-222 inhibition decreases expression of miR-222, Figure S6: Proposed model.

Author Contributions: Conceptualization, N.P., J.T. and K.P.N.; methodology, N.P., R.C. and K.P.N.; validation, N.P., J.T. and W.W.; formal analysis, N.P.; investigation, N.P., J.T., W.W. F.F. and R.S.; resources, R.C.; data curation, N.P., J.T. and W.W.; writing-original draft preparation, N.P., H.M.O., R.C. and K.P.N.; writing—review and editing, N.P., H.M.O., R.C. and K.P.N.; visualization, N.P. and K.P.N.; supervision, N.P. and K.P.N.; project administration, N.P., H.M.O., K.D.M., R.C. and K.P.N.; funding acquisition, N.P. and K.P.N.

Funding: This research was funded by NIH, grant number 5R01CA182832-05.

Acknowledgments: We thank Len Post (former: BioMarin Pharmaceutical Inc.).

Conflicts of Interest: The authors declare no conflict of interest.

\section{References}

1. Girdler, F.; Brotherick, I. The oestrogen receptors (ER alpha and ER beta) and their role in breast cancer: A review. Breast 2000, 9, 194-200. [CrossRef] [PubMed]

2. Lim, E.; Tarulli, G.; Portman, N.; Hickey, T.E.; Tilley, W.D.; Palmieri, C. Pushing estrogen receptor around in breast cancer. Endocr. Relat. Cancer 2016, 23, T227-T241. [CrossRef] [PubMed]

3. Musgrove, E.A.; Sutherland, R.L. Biological determinants of endocrine resistance in breast cancer. Nat. Rev. Cancer 2009, 9, 631-643. [CrossRef] [PubMed]

4. Jin, K.; Park, S.; Teo, W.W.; Korangath, P.; Cho, S.S.; Yoshida, T.; Gyorffy, B.; Goswami, C.P.; Nakshatri, H.; Cruz, L.A.; et al. HOXB7 Is an ERalpha Cofactor in the Activation of HER2 and Multiple ER Target Genes Leading to Endocrine Resistance. Cancer Discov. 2015, 5, 944-959. [CrossRef] [PubMed]

5. Bekele, R.T.; Venkatraman, G.; Liu, R.Z.; Tang, X.; Mi, S.; Benesch, M.G.; Mackey, J.R.; Godbout, R.; Curtis, J.M.; McMullen, T.P.; et al. Oxidative stress contributes to the tamoxifen-induced killing of breast cancer cells: Implications for tamoxifen therapy and resistance. Sci. Rep. 2016, 6, 21164. [CrossRef] [PubMed]

6. Lee, Y.S.; Kang, Y.S.; Lee, S.H.; Kim, J.A. Role of NAD(P)H oxidase in the tamoxifen-induced generation of reactive oxygen species and apoptosis in HepG2 human hepatoblastoma cells. Cell Death Differ. 2000, 7, 925-932. [CrossRef]

7. Kallio, A.; Zheng, A.; Dahllund, J.; Heiskanen, K.M.; Harkonen, P. Role of mitochondria in tamoxifen-induced rapid death of MCF-7 breast cancer cells. Apoptosis 2005, 10, 1395-1410. [CrossRef]

8. Cook, K.L.; Clarke, P.A.; Parmar, J.; Hu, R.; Schwartz-Roberts, J.L.; Abu-Asab, M.; Warri, A.; Baumann, W.T.; Clarke, R. Knockdown of estrogen receptor-alpha induces autophagy and inhibits antiestrogen-mediated unfolded protein response activation, promoting ROS-induced breast cancer cell death. FASEB J. 2014, 28, 3891-3905. [CrossRef]

9. Gutierrez, M.C.; Detre, S.; Johnston, S.; Mohsin, S.K.; Shou, J.; Allred, D.C.; Schiff, R.; Osborne, C.K.; Dowsett, M. Molecular changes in tamoxifen-resistant breast cancer: Relationship between estrogen receptor, HER-2, and p38 mitogen-activated protein kinase. J. Clin. Oncol. 2005, 23, 2469-2476. [CrossRef]

10. Anbalagan, M.; Rowan, B.G. Estrogen receptor alpha phosphorylation and its functional impact in human breast cancer. Mol. Cell. Endocrinol. 2015, 418, 264-272. [CrossRef]

11. Wu, Y.; Zhang, Z.; Cenciarini, M.E.; Proietti, C.J.; Amasino, M.; Hong, T.; Yang, M.; Liao, Y.; Chiang, H.C.; Kaklamani, V.G.; et al. Tamoxifen Resistance in Breast Cancer Is Regulated by the EZH2-ERalpha-GREB1 Transcriptional Axis. Cancer Res. 2018, 78, 671-684. [CrossRef] [PubMed]

12. Badia, E.; Oliva, J.; Balaguer, P.; Cavailles, V. Tamoxifen resistance and epigenetic modifications in breast cancer cell lines. Curr. Med. Chem. 2007, 14, 3035-3045. [CrossRef] [PubMed]

13. Abdel-Hafiz, H.A. Epigenetic Mechanisms of Tamoxifen Resistance in Luminal Breast Cancer. Diseases 2017, 5, 16. [CrossRef] [PubMed]

14. Anbalagan, M.; Huderson, B.; Murphy, L.; Rowan, B.G. Post-translational modifications of nuclear receptors and human disease. Nucl. Recept. Signal. 2012, 10, e001. [CrossRef] [PubMed] 
15. Osborne, C.K.; Schiff, R. Mechanisms of endocrine resistance in breast cancer. Annu. Rev. Med. 2011, 62, $233-247$. [CrossRef]

16. Kim, M.Y.; Zhang, T.; Kraus, W.L. Poly(ADP-ribosyl)ation by PARP-1: 'PAR-laying' NAD+ into a nuclear signal. Genes Dev. 2005, 19, 1951-1967. [CrossRef] [PubMed]

17. Luo, X.; Kraus, W.L. On PAR with PARP: Cellular stress signaling through poly(ADP-ribose) and PARP-1. Genes Dev. 2012, 26, 417-432. [CrossRef]

18. Wright, R.H.; Castellano, G.; Bonet, J.; Le Dily, F.; Font-Mateu, J.; Ballare, C.; Nacht, A.S.; Soronellas, D.; Oliva, B.; Beato, M. CDK2-dependent activation of PARP-1 is required for hormonal gene regulation in breast cancer cells. Genes Dev. 2012, 26, 1972-1983. [CrossRef] [PubMed]

19. Hou, D.; Liu, Z.; Xu, X.; Liu, Q.; Zhang, X.; Kong, B.; Wei, J.J.; Gong, Y.; Shao, C. Increased oxidative stress mediates the antitumor effect of PARP inhibition in ovarian cancer. Redox Biol. 2018, 17, 99-111. [CrossRef] [PubMed]

20. Hou, D.; Xu, G.; Zhang, C.; Li, B.; Qin, J.; Hao, X.; Liu, Q.; Zhang, X.; Liu, J.; Wei, J.; et al. Berberine induces oxidative DNA damage and impairs homologous recombination repair in ovarian cancer cells to confer increased sensitivity to PARP inhibition. Cell Death Dis. 2017, 8, e3070. [CrossRef]

21. Bryant, H.E.; Schultz, N.; Thomas, H.D.; Parker, K.M.; Flower, D.; Lopez, E.; Kyle, S.; Meuth, M.; Curtin, N.J.; Helleday, T. Specific killing of BRCA2-deficient tumours with inhibitors of poly(ADP-ribose) polymerase. Nature 2005, 434, 913-917. [CrossRef]

22. Farmer, H.; McCabe, N.; Lord, C.J.; Tutt, A.N.; Johnson, D.A.; Richardson, T.B.; Santarosa, M.; Dillon, K.J.; Hickson, I.; Knights, C.; et al. Targeting the DNA repair defect in BRCA mutant cells as a therapeutic strategy. Nature 2005, 434, 917-921. [CrossRef] [PubMed]

23. Bitler, B.G.; Watson, Z.L.; Wheeler, L.J.; Behbakht, K. PARP inhibitors: Clinical utility and possibilities of overcoming resistance. Gynecol. Oncol. 2017, 147, 695-704. [CrossRef] [PubMed]

24. Schiewer, M.J.; Knudsen, K.E. Transcriptional roles of PARP1 in cancer. Mol. Cancer Res. 2014, 12, 1069-1080. [CrossRef] [PubMed]

25. Ju, B.G.; Lunyak, V.V.; Perissi, V.; Garcia-Bassets, I.; Rose, D.W.; Glass, C.K.; Rosenfeld, M.G. A topoisomerase Ilbeta-mediated dsDNA break required for regulated transcription. Science 2006, 312, 1798-1802. [CrossRef] [PubMed]

26. Zhang, F.; Wang, Y.; Wang, L.; Luo, X.; Huang, K.; Wang, C.; Du, M.; Liu, F.; Luo, T.; Huang, D.; et al. Poly(ADP-ribose) polymerase 1 is a key regulator of estrogen receptor alpha-dependent gene transcription. J. Biol. Chem. 2013, 288, 11348-11357. [CrossRef]

27. Schiff, R.; Reddy, P.; Ahotupa, M.; Coronado-Heinsohn, E.; Grim, M.; Hilsenbeck, S.G.; Lawrence, R.; Deneke, S.; Herrera, R.; Chamness, G.C.; et al. Oxidative stress and AP-1 activity in tamoxifen-resistant breast tumors in vivo. J. Natl. Cancer Inst. 2000, 92, 1926-1934. [CrossRef]

28. Du, Y.; Yamaguchi, H.; Wei, Y.; Hsu, J.L.; Wang, H.L.; Hsu, Y.H.; Lin, W.C.; Yu, W.H.; Leonard, P.G.; Lee, G.R.T.; et al. Blocking c-Met-mediated PARP1 phosphorylation enhances anti-tumor effects of PARP inhibitors. Nat. Med. 2016, 22, 194-201. [CrossRef]

29. Pulliam, N.; Fang, F.; Ozes, A.R.; Tang, J.; Adewuyi, A.; Keer, H.N.; Lyons, J.F.; Baylin, S.B.; Matei, D.; Nakshatri, H.; et al. An Effective Epigenetic-PARP inhibitor Combination Therapy for Breast and Ovarian Cancers Independent of BRCA-mutations. Clin. Cancer Res. 2018. [CrossRef]

30. Fan, M.; Yan, P.S.; Hartman-Frey, C.; Chen, L.; Paik, H.; Oyer, S.L.; Salisbury, J.D.; Cheng, A.S.; Li, L.; Abbosh, P.H.; et al. Diverse gene expression and DNA methylation profiles correlate with differential adaptation of breast cancer cells to the antiestrogens tamoxifen and fulvestrant. Cancer Res 2006, 66, 11954-11966. [CrossRef]

31. Chou, T.C.; Talalay, P. Quantitative analysis of dose-effect relationships: The combined effects of multiple drugs or enzyme inhibitors. Adv. Enzyme Regul. 1984, 22, 27-55. [CrossRef]

32. Lord, C.J.; Ashworth, A. PARP inhibitors: Synthetic lethality in the clinic. Science 2017, 355, 1152-1158. [CrossRef] [PubMed]

33. Brunner, N.; Boysen, B.; Jirus, S.; Skaar, T.C.; Holst-Hansen, C.; Lippman, J.; Frandsen, T.; Spang-Thomsen, M.; Fuqua, S.A.; Clarke, R. MCF7/LCC9: An antiestrogen-resistant MCF-7 variant in which acquired resistance to the steroidal antiestrogen ICI 182,780 confers an early cross-resistance to the nonsteroidal antiestrogen tamoxifen. Cancer Res. 1997, 57, 3486-3493. [PubMed]

34. Kocanova, S.; Mazaheri, M.; Caze-Subra, S.; Bystricky, K. Ligands specify estrogen receptor alpha nuclear localization and degradation. BMC Cell Biol. 2010, 11, 98. [CrossRef] [PubMed] 
35. Razandi, M.; Oh, P.; Pedram, A.; Schnitzer, J.; Levin, E.R. ERs associate with and regulate the production of caveolin: Implications for signaling and cellular actions. Mol. Endocrinol. 2002, 16, 100-115. [CrossRef] [PubMed]

36. Sakai, W.; Swisher, E.M.; Karlan, B.Y.; Agarwal, M.K.; Higgins, J.; Friedman, C.; Villegas, E.; Jacquemont, C.; Farrugia, D.J.; Couch, F.J.; et al. Secondary mutations as a mechanism of cisplatin resistance in BRCA2-mutated cancers. Nature 2008, 451, 1116-1120. [CrossRef] [PubMed]

37. Qi, H.; Jiang, Z.; Wang, C.; Yang, Y.; Li, L.; He, H.; Yu, Z. Sensitization of tamoxifen-resistant breast cancer cells by Z-ligustilide through inhibiting autophagy and accumulating DNA damages. Oncotarget 2017, 8, 29300-29317. [CrossRef] [PubMed]

38. Neijenhuis, S.; Bajrami, I.; Miller, R.; Lord, C.J.; Ashworth, A. Identification of miRNA modulators to PARP inhibitor response. DNA Repair 2013, 12, 394-402. [CrossRef]

39. Rao, X.; Di Leva, G.; Li, M.; Fang, F.; Devlin, C.; Hartman-Frey, C.; Burow, M.E.; Ivan, M.; Croce, C.M.; Nephew, K.P. MicroRNA-221/222 confers breast cancer fulvestrant resistance by regulating multiple signaling pathways. Oncogene 2011, 30, 1082-1097. [CrossRef]

40. Di Leva, G.; Gasparini, P.; Piovan, C.; Ngankeu, A.; Garofalo, M.; Taccioli, C.; Iorio, M.V.; Li, M.; Volinia, S.; Alder, H.; et al. MicroRNA cluster 221-222 and estrogen receptor alpha interactions in breast cancer. J. Natl. Cancer Inst. 2010, 102, 706-721. [CrossRef]

41. Manavalan, T.T.; Teng, Y.; Appana, S.N.; Datta, S.; Kalbfleisch, T.S.; Li, Y.; Klinge, C.M. Differential expression of microRNA expression in tamoxifen-sensitive MCF-7 versus tamoxifen-resistant LY2 human breast cancer cells. Cancer Lett. 2011, 313, 26-43. [CrossRef] [PubMed]

42. Early Breast Cancer Trialists' Collaborative Group. Long-term outcomes for neoadjuvant versus adjuvant chemotherapy in early breast cancer: Meta-analysis of individual patient data from ten randomised trials. Lancet Oncol. 2018, 19, 27-39. [CrossRef]

43. Dowsett, M.; Nicholson, R.I.; Pietras, R.J. Biological characteristics of the pure antiestrogen fulvestrant: Overcoming endocrine resistance. Breast Cancer Res. Treat. 2005, 93, S11-S18. [CrossRef] [PubMed]

44. Jordan, V.C. Tamoxifen Resistance Trumped and Oral Selective Estrogen Receptor Degraders Arrive. Clin. Cancer Res. 2018. [CrossRef] [PubMed]

45. Trachootham, D.; Alexandre, J.; Huang, P. Targeting cancer cells by ROS-mediated mechanisms: A radical therapeutic approach? Nat. Rev. Drug Discov. 2009, 8, 579-591. [CrossRef] [PubMed]

46. Muvarak, N.E.; Chowdhury, K.; Xia, L.; Robert, C.; Choi, E.Y.; Cai, Y.; Bellani, M.; Zou, Y.; Singh, Z.N.; Duong, V.H.; et al. Enhancing the cytotoxic effects of PARP inhibitors with DNA demethylating agents-A potential therapy for cancer. Cancer Cell 2016, 30, 637-650. [CrossRef] [PubMed]

47. Ozes, A.R.; Miller, D.F.; Ozes, O.N.; Fang, F.; Liu, Y.; Matei, D.; Huang, T.; Nephew, K.P. NF-kappaB-HOTAIR axis links DNA damage response, chemoresistance and cellular senescence in ovarian cancer. Oncogene 2016, 35, 5350-5361. [CrossRef] [PubMed]

48. Ozes, A.R.; Pulliam, N.; Ertosun, M.G.; Yilmaz, O.; Tang, J.; Copuroglu, E.; Matei, D.; Ozes, O.N.; Nephew, K.P. Protein kinase A-mediated phosphorylation regulates STAT3 activation and oncogenic EZH2 activity. Oncogene 2018. [CrossRef]

49. Sakai, W.; Swisher, E.M.; Jacquemont, C.; Chandramohan, K.V.; Couch, F.J.; Langdon, S.P.; Wurz, K.; Higgins, J.; Villegas, E.; Taniguchi, T. Functional restoration of BRCA2 protein by secondary BRCA2 mutations in BRCA2-mutated ovarian carcinoma. Cancer Res. 2009, 69, 6381-6386. [CrossRef]

50. Available online: http://www.combosyn.com/feature.html (accessed on 4 January 2019).

(C) 2019 by the authors. Licensee MDPI, Basel, Switzerland. This article is an open access article distributed under the terms and conditions of the Creative Commons Attribution (CC BY) license (http://creativecommons.org/licenses/by/4.0/). 